\title{
On the dynamics of a class of rational Kolmogorov systems
}

Rachid Boukoucha, Ahmed Bendjeddou

To cite this article: Rachid Boukoucha, Ahmed Bendjeddou (2016) On the dynamics of a class of rational Kolmogorov systems, Journal of Nonlinear Mathematical Physics 23:1, 21-27, DOI: https://doi.org/10.1080/14029251.2016.1135629

To link to this article: https://doi.org/10.1080/14029251.2016.1135629

Published online: 04 January 2021 


\title{
On the dynamics of a class of rational Kolmogorov systems
}

\author{
Rachid Boukoucha \\ Department of Mathematics, Faculty of Exact Sciences and \\ Informatics, University of Jijel, 18000 Jijel, Algeria \\ rachid_boukecha@yahoo.fr \\ Ahmed Bendjeddou \\ Departement de Mathematics, Faculty of Sciences, University \\ of Setif, 19000 Setif, Algeria \\ bendjeddou@univ-setif.dz
}

Received 25 August 2015

Accepted 22 September 2015

In this paper we are intersted in studying the existence of a First integral and the non-existence of limit cycles of rational Kolmogorov systems of the form

$$
\left\{\begin{array}{l}
x^{\prime}=x\left(P(x, y)+\frac{R(x, y)}{S(x, y)}\right), \\
y^{\prime}=y\left(Q(x, y)+\frac{R(x, y)}{S(x, y)}\right),
\end{array}\right.
$$

where $P(x, y), Q(x, y), R(x, y), S(x, y)$ are homogeneous polynomials of degree $n, n, m, a$ respectively.

Keywords: Kolmogorov System, First Integral, Periodic Orbits, Limit Cycle.

2010 Mathematics Subject Classification: 34C05, 34C07, 37C27, 37K10.

\section{Introduction}

The autonomous differential system on the plane given by

$$
\left\{\begin{array}{l}
x^{\prime}=\frac{d x}{d t}=x F(x, y), \\
y^{\prime}=\frac{d y}{d t}=y G(x, y),
\end{array}\right.
$$

is known as Kolmogorov system, the derivatives are performed with respect to the time variable, and $F, G$ are two functions in the variables $x$ and $y$. Is frequently used to model the interation of two species occupying the same ecological niche, see $[11,15,18]$. There are many natural phenomena which can be modeled by the Kolmogorov systems such as mathematical ecology and population dynamics see $[13,19,20]$ chemical reactions, plasma physics see [14], hydrodynamics see [6], economics, etc. In the classical Lotka- Volterra-Gause model, $F$ and $G$ are linear and it is well known that there are no limit cycles. There can, of course, only be one critical point in the interior of the realistic quadrant $(x>0, y>0)$ in this case, but this can be a center; however, there are no isolated periodic solutions. We remind that in the phase plane, a limit cycle of system (1.1) is an isolated periodic orbit in the set of all periodic orbits of system (1.1). 
In the qualitative theory of planar dynamical systems see $[5,8,9,10,16,17]$, one of the most important topics is related to the second part of the unsolved Hilbert 16th problem. There is a huge literature about limit cycles, most of them deal essentially with their detection, their number and their stability and rare are papers concerned by giving them explicitly see $[1,2,3,4,12]$.

Let $\Omega$ be a non-empty open and dense subset of $\mathbb{R}^{2}$. We say that a non-locally constant $C^{1}$ function $H: \Omega \rightarrow \mathbb{R}$ is a first integral of the differential system (1.1) in $\Omega$ if $H$ is constant on the trajectories of the system (1.1) contained in $\Omega$, i.e. if

$$
\frac{d H(x, y)}{d t}=\frac{\partial H(x, y)}{\partial x} x F(x, y)+\frac{\partial H(x, y)}{\partial y} y G(x, y) \equiv 0 \text { in the points of } \Omega .
$$

Moreover, $H=h$ is the general solution of this equation, where $h$ is an arbitrary constant. It is well known that for differential systems defined on the plane $\mathbb{R}^{2}$ the existence of a first integral determines their phase portrait see [7].

In this paper we are intersted in studying the existence of a First integral and the non-existence of limit cycles of the 2-dimensional rational Kolmogorov systems of the form

$$
\left\{\begin{array}{l}
x^{\prime}=x\left(P(x, y)+\frac{R(x, y)}{S(x, y)}\right), \\
y^{\prime}=y\left(Q(x, y)+\frac{R(x, y)}{S(x, y)}\right),
\end{array}\right.
$$

where $P(x, y), Q(x, y), R(x, y), S(x, y)$ are homogeneous polynomials of degree $n, n, m, a$ respectively.

We define the trigonometric functions

$$
\begin{aligned}
& f_{1}(\theta)=P(\cos \theta, \sin \theta) \cos ^{2} \theta+Q(\cos \theta, \sin \theta) \sin ^{2} \theta, f_{2}(\theta)=\frac{R(\cos \theta \sin \theta)}{S(\cos \theta, \sin \theta)}, \\
& f_{3}(\theta)=Q(\cos \theta, \sin \theta) \cos \theta \sin \theta-P(\cos \theta, \sin \theta) \cos \theta \sin \theta .
\end{aligned}
$$

\section{Main result}

Our main result on the existence of a First integral and the periodic orbits of the rational Kolmogorov system (1.2) is the following.

Theorem 2.1. Consider a rational Komogorov system (1.2). Then the following statements hold.

(a) if $f_{3}(\theta) \neq 0, S(\cos \theta, \sin \theta) \neq 0$ and $n+a \neq m$, then system (1.2) has the first integral

$$
\begin{aligned}
H(x, y)= & \left(x^{2}+y^{2}\right)^{\frac{n-m+a}{2}} \exp \left((m-n-a) \int^{\arctan \frac{y}{x}} A(\omega) d \omega\right)- \\
& (n-m+a) \int^{\arctan \frac{y}{x}} \exp \left((m-n-a) \int^{w} A(\omega) d \omega\right) B(w) d w,
\end{aligned}
$$

where $A(\theta)=\frac{f_{1}(\theta)}{f_{3}(\theta)}, B(\theta)=\frac{f_{2}(\theta)}{f_{3}(\theta)}$, and the curves which are formed by the trajectories of the differential system (1.2), are written in Cartesian coordinates as

$$
x^{2}+y^{2}=\left(\begin{array}{c}
h \exp \left((n-m+a) \int^{\arctan \frac{y}{x}} A(\omega) d \omega\right)+ \\
(n-m+a) \exp \left((n-m+a) \int^{\theta} A(\omega) d \omega\right) \\
\int^{\arctan \frac{y}{x}} \exp \left((m-n-a) \int^{w} A(\omega) d \omega\right) B(w) d w
\end{array}\right)^{\frac{2}{n-m+a}}
$$

where $h \in \mathbb{R}$. Moreover, the system (1.2) has no limit cycle. 
(b) if $f_{3}(\theta) \neq 0, S(\cos \theta, \sin \theta) \neq 0$ and $n+a=m$, then system (1.2) has the first integral

$$
H(x, y)=\left(x^{2}+y^{2}\right)^{\frac{1}{2}} \exp \left(-\int^{\arctan \frac{y}{x}}(A(\omega)+B(\omega)) d \omega\right),
$$

and the curves which are formed by the trajectories of the differential system (1.2), are written in Cartesian coordinates as

$$
\left(x^{2}+y^{2}\right)^{\frac{1}{2}}-h \exp \left(\int^{\arctan \frac{y}{x}}(A(\omega)+B(\omega)) d \omega\right)=0,
$$

where $h \in \mathbb{R}$. Moreover, the system (1.2) has no limit cycle.

(c) if $f_{3}(\theta)=0$ for all $\theta \in \mathbb{R}$, then system (1.2) has the first integral $H=\frac{y}{x}$, and the curves which are formed by the trajectories of the differential system (1.2), are written in Cartesian coordinates as $y=h x$ where $h \in \mathbb{R}$. Moreover, system (1.2) has no limit cycle.

Proof. In order to prove our results we write the rational differential system (1.2) in polar coordinates $(r, \theta)$, defined by $x=r \cos \theta$, and $y=r \sin \theta$, then system (1.2) becomes

$$
\left\{\begin{array}{l}
r^{\prime}=f_{1}(\theta) r^{n+1}+f_{2}(\theta) r^{m-a+1} \\
\theta^{\prime}=f_{3}(\theta) r^{n}
\end{array}\right.
$$

where the trigonometric functions $f_{1}(\theta), f_{2}(\theta)$ and $f_{3}(\theta)$ already given in introduction, $r^{\prime}=\frac{d r}{d t}$ and $\theta^{\prime}=\frac{d \theta}{d t}$.

If $f_{3}(\theta) \neq 0$ and $n+a \neq m$ : Taking as independent variable the coordinate $\theta$, this differential system (2.1) writes as the Bernoulli equation

$$
\frac{d r}{d \theta}=A(\theta) r+B(\theta) r^{1+m-n-a},
$$

where $A(\theta)=\frac{f_{1}(\theta)}{f_{3}(\theta)}$ and $B(\theta)=\frac{f_{2}(\theta)}{f_{3}(\theta)}$.

By introducing the standard change of variables $\rho=r^{n-m+a}$, we obtain the linear equation

$$
\frac{d \rho}{d \theta}=(n-m+a)(A(\theta) \rho+B(\theta)) .
$$

The general solution of linear equation $(2.3)$ is

$$
\begin{aligned}
\rho(\theta)= & \exp \left((n-m+a) \int^{\theta} A(\omega) d \omega\right) \\
& \left(\alpha+(n-m+a) \int^{\theta} \exp \left((m-n-a) \int^{w} A(\omega) d \omega\right) B(w) d w\right),
\end{aligned}
$$

where $\alpha \in \mathbb{R}$, which has the first integral

$$
\begin{aligned}
H(x, y)= & \left(x^{2}+y^{2}\right)^{\frac{n-m+a}{2}} \exp \left((m-n-a) \int^{\arctan \frac{y}{x}} A(\omega) d \omega\right)- \\
& (n-m+a) \int^{\arctan \frac{y}{x}} \exp \left((m-n-a) \int^{w} A(\omega) d \omega\right) B(w) d w .
\end{aligned}
$$

Let $\gamma$ be a periodic orbit surrounding an equilibrium located in the realistic quadrant $(x>0, y>0)$, and let $h_{\gamma}=H(\gamma)$. 
The curves $H=h$ with $h \in \mathbb{R}$, which are formed by trajectories of the differential system (1.2), are written as

$$
r(\theta)=\left(\begin{array}{c}
h \exp \left((n-m+a) \int^{\theta} A(\omega) d \omega\right)+ \\
(n-m+a) \exp \left((n-m+a) \int^{\theta} A(\omega) d \omega\right) \\
\int^{\theta} \exp \left((m-n-a) \int^{w} A(\omega) d \omega\right) B(w) d w
\end{array}\right)^{\frac{1}{n-m+a}}
$$

In Cartesian coordinates $r^{2}=x^{2}+y^{2}$ and $\theta=\arctan \left(\frac{y}{x}\right)$, we have

$$
x^{2}+y^{2}=\left(\begin{array}{c}
h \exp \left((n-m+a) \int^{\arctan \frac{y}{x}} A(\omega) d \omega\right)+ \\
(n-m+a) \exp \left((n-m+a) \int^{\theta} A(\omega) d \omega\right) \\
\int^{\arctan \frac{y}{x}} \exp \left((m-n-a) \int^{w} A(\omega) d \omega\right) B(w) d w
\end{array}\right)^{\frac{2}{n-m+a}}
$$

Therefore the periodic orbit $\gamma$ is contained in the curve

$$
r(\theta)=\left(\begin{array}{c}
h_{\gamma} \exp \left((n-m+a) \int^{\theta} A(\omega) d \omega\right)+ \\
(n-m+a) \exp \left((n-m+a) \int^{\theta} A(\omega) d \omega\right) \\
\int^{\theta} \exp \left((m-n-a) \int^{w} A(\omega) d \omega\right) B(w) d w
\end{array}\right)^{\frac{1}{n-m+a}}
$$

But this curve cannot contain the periodic orbit $\gamma$, consequently no limit cycle is contained in the realistic quadrant $(x>0, y>0)$, because this curve at most have a unique point on every ray $\theta=\theta^{*}$ for all $\theta^{*} \in[0,2 \pi)$. Hence statement $(a)$ of Theorem 1 is proved.

Suppose now that $f_{3}(\theta) \neq 0$, and $n+a=m$. Taking as independent variable the coordinate $\theta$, this differential system $(2.1)$ writes

$$
\frac{d r}{d \theta}=(A(\theta)+B(\theta)) r
$$

The general solution of equation (2.4) is

$$
r(\theta)=\alpha \exp \left(\int^{\theta}(A(\omega)+B(\omega)) d \omega\right)
$$

where $\alpha \in \mathbb{R}$, which has the first integral

$$
H(x, y)=\left(x^{2}+y^{2}\right)^{\frac{1}{2}} \exp \left(-\int^{\arctan \frac{y}{x}}(A(\omega)+B(\omega)) d \omega\right)
$$

Let $\gamma$ be a periodic orbit surrounding an equilibrium located in the realistic quadrant $(x>0, y>0)$, and let $h_{\gamma}=H(\gamma)$. The curves $H=h$ with $h \in \mathbb{R}$, which are formed by trajectories of the differential 
system (1.2), are written as

$$
r(\theta)=h \exp \left(\int^{\theta}(A(\omega)+B(\omega)) d \omega\right)
$$

In Cartesian coordinates $r^{2}=x^{2}+y^{2}$ and $\theta=\arctan \left(\frac{y}{x}\right)$, we have

$$
\left(x^{2}+y^{2}\right)^{\frac{1}{2}}-h \exp \left(\int^{\arctan \frac{y}{x}}(A(\omega)+B(\omega)) d \omega\right)=0 .
$$

Therefore the periodic orbit $\gamma$ is contained in the curve

$$
r(\theta)=h_{\gamma} \exp \left(\int^{\arctan \frac{y}{x}}(A(\omega)+B(\omega)) d \omega\right) .
$$

But this curve cannot contain the periodic orbit $\gamma$, consequently no limit cycle is contained in the realistic quadrant $(x>0, y>0)$, because this curve at most have a unique point on every ray $\theta=\theta^{*}$ for all $\theta^{*} \in[0,2 \pi)$. Hence statement $(b)$ of Theorem 1 is proved.

Assume now that $f_{3}(\theta)=0$ for all $\theta \in \mathbb{R}$, then from (2.1) it follows that $\theta^{\prime}=0$. So the straight lines through the origin of coordinates of the differential system (1.2) are invariant by the flow of this system. Hence, $\frac{y}{x}$ is a first integral of the system(1.2), since all the straight lines through the origin are formed by trajectories. Then the curves $H=h$ with $h \in \mathbb{R}$, which are formed by trajectories of the differential system (1.2), in Cartesian coordinates written as $y=h x$ where $h \in \mathbb{R}$. Clearly the system (1.2) has no periodic orbits, consequently no limit cycle.

This completes the proof of statement $(c)$ of Theorem 1.

\section{Examples}

The following examples are given to illustrate our result.

Example 1. If we take $P(x, y)=2 x y-y^{2}, Q(x, y)=2 x y+x^{2}, R(x, y)=x-y$ and $S(x, y)=x+y$, then system (1.2) reads

$$
\left\{\begin{array}{l}
x^{\prime}=x\left(2 x y-y^{2}+\frac{x-y}{x+y}\right), \\
y^{\prime}=y\left(x^{2}+2 x y+\frac{x-y}{x+y}\right) .
\end{array}\right.
$$

The rational differential system (3.1) in Polar coordinates $(r, \theta)$ becomes

$$
\left\{\begin{array}{l}
r^{\prime}=(\sin 2 \theta) r^{3}+\frac{\cos \theta-\sin \theta}{\cos \theta+\sin \theta} r \\
\theta^{\prime}=\frac{1}{2}(\sin 2 \theta) r^{2}
\end{array}\right.
$$

here $f_{1}(\theta)=\sin 2 \theta, f_{2}(\theta)=\frac{\cos \theta-\sin \theta}{\cos \theta+\sin \theta}$ and $f_{3}(\theta)=\frac{1}{2} \sin 2 \theta$. In the realistic quadrant $(x>0, y>0)$ it is the case $(a)$ of the Theorem 1, then the rational differential system (3.1) has the first integral

$$
\begin{aligned}
H(x, y)= & \left(x^{2}+y^{2}\right) \exp \left(-4 \arctan \frac{y}{x}\right)- \\
& \int^{\arctan \frac{y}{x}} \exp (-4 w)\left(\frac{4 \cos w-4 \sin w}{(\cos w+\sin w) \sin 2 w}\right) d w .
\end{aligned}
$$


The curves $H=h$ with $h \in \mathbb{R}$, which are formed by trajectories of the differential system (3.1), in Cartesian coordinates are written as

$$
\begin{aligned}
x^{2}+y^{2}= & h \exp \left(4 \arctan \frac{y}{x}\right)+ \\
& \exp \left(4 \arctan \frac{y}{x}\right) \int^{\arctan \frac{y}{x}} \exp (-4 w)\left(\frac{4 \cos w-4 \sin w}{(\cos w+\sin w) \sin 2 w}\right) d w,
\end{aligned}
$$

where $h \in \mathbb{R}$. Moreover, the system (3.1) has no limit cycle.

Example 2. If we take $P(x, y)=x+y, Q(x, y)=x+2 y, R(x, y)=-2 y^{3}-x^{3}+5 x^{2} y+8 x y^{2}$ and $S(x, y)=x^{2}+y^{2}$, then system $(1.2)$ reads

$$
\left\{\begin{array}{l}
x^{\prime}=x\left(x+y+\frac{-2 y^{3}-x^{3}+5 x^{2} y+8 x y^{2}}{x^{2}+y^{2}}\right) \\
y^{\prime}=y\left(x+2 y+\frac{-2 y^{3}-x^{3}+5 x^{2} y+8 x y^{2}}{x^{2}+y^{2}}\right) .
\end{array}\right.
$$

The rational differential system (3.2) in Polar coordinates $(r, \theta)$ becomes

$$
\left\{\begin{array}{l}
r^{\prime}=\left(9 \cos \theta \sin ^{2} \theta+6 \cos ^{2} \theta \sin \theta\right) r^{2} \\
\theta^{\prime}=(\cos \theta)\left(\sin ^{2} \theta\right) r
\end{array}\right.
$$

here $f_{1}(\theta)=\cos \theta+\frac{7}{4} \sin \theta-\frac{1}{4} \sin 3 \theta, f_{2}(\theta)=-2 \sin ^{3} \theta-\cos ^{3} \theta+5 \cos ^{2} \theta \sin \theta+8 \cos \theta \sin ^{2} \theta$, $f_{3}(\theta)=\frac{1}{4} \cos \theta-\frac{1}{4} \cos 3 \theta$. In the realistic quadrant $(x>0, y>0)$ it is the case $(b)$ of the Theorem 1 , the rational differential system (3.2) has the first integral

$$
H(x, y)=\left(x^{2}+y^{2}\right)^{\frac{1}{2}} \exp \left(-\int^{\arctan \frac{y}{x}}\left(\frac{9 \sin w+6 \cos w}{\sin w}\right) d w\right) .
$$

The curves $H=h$ with $h \in \mathbb{R}$, which are formed by trajectories of the differential system (3.2), in Cartesian coordinates are written as

$$
\left(x^{2}+y^{2}\right)^{\frac{1}{2}}=h \exp \left(\int \arctan \frac{y}{x}\left(\frac{9 \sin w+6 \cos w}{\sin w}\right) d w\right),
$$

where $h \in \mathbb{R}$. Moreover, the system (3.2) has no limit cycle.

Example 3. If we take $P(x, y)=x+y, Q(x, y)=x+y, R(x, y)=2 x+3 y$ and $S(x, y)=x^{2}+y^{2}$, then system (1.2) reads

$$
\left\{\begin{array}{l}
x^{\prime}=x\left(x+y+\frac{2 x+3 y}{x^{2}+y^{2}}\right), \\
y^{\prime}=y\left(x+y+\frac{2 x+3 y}{x^{2}+y^{2}}\right) .
\end{array}\right.
$$

The rational differential system (3.3) in Polar coordinates $(r, \theta)$ becomes

$$
\left\{\begin{array}{l}
r^{\prime}=2 \cos \theta+3 \sin \theta+(\cos \theta+\sin \theta) r^{2}, \\
\theta^{\prime}=0,
\end{array}\right.
$$

here $f_{1}(\theta)=\cos \theta+\sin \theta, f_{2}(\theta)=2 \cos \theta+3 \sin \theta, f_{3}(\theta)=0$ for all $\theta \in \mathbb{R}$, so it is the case (c) of the Theorem 1. Hence, $\frac{y}{x}$ is a first integral of the system (3.3). Then the curves $H=h$ with $h \in \mathbb{R}$, which are formed by trajectories of the differential system (3.3), are written in Cartesian 
coordinates as $y=h x$ where $h \in \mathbb{R}$. Clearly the system (3.3)has no periodic orbits, consequently no limit cycle.

\section{Conclusion}

The elementary method used in this paper seems to be fruitful to investigate more general planar rational differential systems of ODEs in order to obtain explicit expression for a first integral which characterizes its trajectories. This is one of the classical tools in the classification of all trajectories of dynamical systems.

\section{References}

[1] A. Bendjeddou and R. Boukoucha; Explict non-algebraic limit cycles of a class of polynomial systems, FJAM Volume 91 No.2 (2015) 133-142.

[2] A. Bendjeddou and R. Cheurfa; Cubic and quartic planar differential system with exact algebraic limit cycles, Electronic Journal of Differential Equations (EJDO). 2011(ejde.math.txstate.edu/ ).

[3] A. Bendjeddou, J. Llibre, T. Salhi, Dynamics of the differential systems with homogenous nonlinearities and a star node, J. Differential Equations 254 (2013) 3530-3537.

[4] R. Boukoucha and A. Bendjeddou; A Quintic polynomial diffential systems with explicit non-algebraic limit cycle, International Journal of Pure and Applied Mathematics Volume No. 2 2015, 235-241.

[5] R. Boukoucha and A. Bendjeddou; On the non-existence of limit cycles for a cubic kolmogorov systems, International Journal of Pure and Applied Mathematics Volume No. 2 2015, 227-233.

[6] F. H. Busse, Transition to turbulence via the statistical limit cycle route, Synergetics, Springer-Verlag, berlin,1978. p.39.

[7] L. Cairó, J. Llibre, Phase portraits of cubic polynomial vector fields of Lotka-Volterra type having a rational first integral of degree 2, J. Phys. A 40 (2007) 6329-6348.]

[8] J. Chavarriga and I A. Garc'ia, Existence of limit cycles for real quadratic differential systems with an invariant cubic, Pacific Journal of Mathematics, Volume 223 No.2 201-218 (2006).

[9] A D. Khalil I. T, Non-algebraic limit cycles for parameterized planar polynomial systems, Int. J. Math 18, No. 2, 179-189 (2007).

[10] F. Dumortier, J. Llibre and J. Artés, Qualitative Theory of Planar Differential Systems, (Universitex) Berlin, Springer (2006).

[11] P. Gao, Hamiltonian structure and first integrals for the Lotka-Volterra systems, Phys. Lett. A 273 (2000) 85-96.

[12] A. Gasull, H. Giacomini and J. Torregrosa, Explicit non-algebraic limit cycles for polynomial systems, J. Comput. Appl. Math. 200 (2007), 448-457.

[13] X. Huang, Limit in a Kolmogorov-type Moel, Internat. J. Math. and Math Sci. Vol. 13 No. 3 (1990) 555-566.

[14] G. Lavel, R. Pellat, Pellat, Plasma Physics, in: Proceedings of Summer School of Theoreal Physics, Gordon and Breach, New York, 1975.

[15] C. Li, J. Llibre, The cyclicity of period annulus of a quadratic reversible Lotka-Volterra system, Nonlinearity 22 (2009) 2971-2979.

[16] J. Llibre, T. Salhi, On the dynamics of class of Kolmogorov systems, J. Appl. Math.and Comput 225 (2013), 242-245.

[17] J. Llibre, J. Yu, X. Zhang, On the limit Cycle of the Polynomial Differential Systems with a Linear Node and Homogeneous Nonlinearities, International of Bifurcation and Choos, Vol. 24, No. 5 (2014) 1450065 (7pages).

[18] J. Llibre, C. Valls, Polynomial, rational and analytic first integrals for a family of 3-dimensional LotkaVolterra systems, Z. Angew. Math. Phys. 62 (2011) 761-777.

[19] N. G. Llyod and J. M. Pearson, Limit cycles of a Cubic Kolmogorov System, Appl. Math. Lett. Vol. 9, 1, pp, 15-18, 1996.

[20] R.M. May, Stability and complexity in Model Ecosystems, Princeton, New Jersey, 1974. 